\title{
Oxygen Electromigration and Energy Band Reconstruction Induced by Electrolyte Field Effect at Oxide Interfaces
}

\author{
S. W. Zeng, ${ }^{1,2, *}$ X. M. Yin, ${ }^{2,3,4}$ T. S. Herng, ${ }^{5}$ K. Han, ${ }^{1,2}$ Z. Huang, ${ }^{1,2}$ L. C. Zhang, ${ }^{1,2}$ \\ C. J. Li, ${ }^{1,5}$ W. X. Zhou, ${ }^{1,2}$ D. Y. Wan, ${ }^{1,2}$ P. Yang, ${ }^{3}$ J. Ding, ${ }^{1,5}$ A. T. S. Wee, ${ }^{2,6}$ \\ J. M. D. Coey, ${ }^{1,7}$ T. Venkatesan, ${ }^{1,2,5,8,9}$ A. Rusydi, ${ }^{1,2,3}$ and A. Ariando ${ }^{1,2,9, \dagger}$ \\ ${ }^{1}$ NUSNNI-NanoCore, National University of Singapore, Singapore 117411, Singapore \\ ${ }^{2}$ Department of Physics, National University of Singapore, Singapore 117542, Singapore \\ ${ }^{3}$ Singapore Synchrotron Light Source (SSLS), National University of Singapore, 5 Research Link, Singapore 117603, Singapore \\ ${ }^{4}$ SZU-NUS Collaborative Innovation Center for Optoelectronic Science \& Technology, \\ Key Laboratory of Optoelectronic Devices and Systems of Ministry of Education and Guangdong Province, \\ College of Optoelectronic Engineering, Shenzhen University, Shenzhen 518060, China \\ ${ }^{5}$ Department of Materials Science and Engineering, National University of Singapore, Singapore 117576, Singapore \\ ${ }^{6}$ Centre for Advanced 2D Materials and Graphene Research, National University of Singapore, Singapore 117546, Singapore \\ ${ }^{7}$ School of Physics and CRANN, Trinity College, Dublin 2, Ireland \\ ${ }^{8}$ Department of Electrical and Computer Engineering, National University of Singapore, Singapore 117576, Singapore \\ ${ }^{9}$ National University of Singapore Graduate School for Integrative Sciences and Engineering (NGS), \\ 28 Medical Drive, Singapore 117456, Singapore
}

(Received 28 September 2017; published 5 October 2018)

\begin{abstract}
Electrolyte gating is a powerful means for tuning the carrier density and exploring the resultant modulation of novel properties on solid surfaces. However, the mechanism, especially its effect on the oxygen migration and electrostatic charging at the oxide heterostructures, is still unclear. Here we explore the electrolyte gating on oxygen-deficient interfaces between $\mathrm{SrTiO}_{3}$ (STO) crystals and $\mathrm{LaAlO}_{3}(\mathrm{LAO})$ overlayer through the measurements of electrical transport, x-ray absorption spectroscopy, and photoluminescence spectra. We found that oxygen vacancies $\left(\mathrm{O}_{\mathrm{vac}}\right)$ were filled selectively and irreversibly after gating due to oxygen electromigration at the amorphous LAO/STO interface, resulting in a reconstruction of its interfacial band structure. Because of the filling of $\mathrm{O}_{\mathrm{vac}}$, the amorphous interface also showed an enhanced electron mobility and quantum oscillation of the conductance. Further, the filling effect could be controlled by the degree of the crystallinity of the LAO overlayer by varying the growth temperatures. Our results reveal the different effects induced by electrolyte gating, providing further clues to understand the mechanism of electrolyte gating on buried interfaces and also opening a new avenue for constructing highmobility oxide interfaces.
\end{abstract}

DOI: 10.1103/PhysRevLett.121.146802

Electric field effect doping, which is the foundation of modern semiconductor electronics, has been a flexible and powerful technique for tuning the carrier density and the resultant transport properties of a material [1]. More recently, the electric field provided by electronic double layer transistors (EDLTs) with ionic liquids (ILs) or polymer electrolytes as the gate dielectrics has enabled the accumulation of a large amount of charge carriers up to $\sim 10^{15} / \mathrm{cm}^{2}$ [2], and, consequently, novel phase transitions in a variety of materials have been induced [3-16]. In an EDLT, the induced charged layer on the sample surface may be a direct result of electrostatic carrier injection [3-11,13,14,17], migration of oxygen [15,16,18-26] and hydrogen [16,27-29], and/or structural changes [30-33]. Specifically, for the oxygen-free two-dimensional (2D) layered materials, the dominant driving mechanism has been shown to be electrostatic charging, and thus allowing the study of ion gating of clean 2D superconductors [4,6-11]. In complex oxides, however, oxygen migration is possible due to the electrochemical reaction on the sample surface and the large electric field as the IL is directly applied on the oxide surface $[15,18,19]$. Through insertion of a chemically inert layer between the IL and conducting channel, the oxides can be protected against electrochemical reactions, leading to an enhancement of the carrier mobility [34-36]. However, the gating mechanism, especially the effect of oxygen electromigration on buried oxides under the protective overlayer and the related influence on the band structure is still unclear [34-38].

STO-based oxide heterostructures such as the interface with LAO overlayer are of great interest [39-45]. Tunability of electrical properties at the $\mathrm{LaAlO}_{3} / \mathrm{SrTiO}_{3}$ (LAO/STO) interface could be realized using various methods such as field effect [42], metal capping [46], surface adsorbates [45], and defect control [47], suggesting 
its potential for oxide-based electronics [48,49]. Conducting interfaces can be even constructed at room temperature by depositing amorphous films on crystalline STO, which have the advantage of being compatible with established semiconductor fabrication processes [50-52]. Moreover, high carrier mobility at the amorphous LAO/STO $(a$-LAO/STO $)$ interface is achieved by charge-transfer-induced modulation doping, through inserting a $\mathrm{La}_{1-x} \mathrm{Sr}_{x} \mathrm{MnO}_{3}$ layer as the potential barrier, suggesting the importance of interfacial band structure for charge transport [53]. In this Letter, we demonstrate IL gating induced oxygen electromigration on LAO/STO interfaces and the resultant change of band structure due to the selective filling of interfacial $\mathrm{O}_{\text {vac }}$.

The interfaces were obtained by depositing LAO on STO substrates using a pulsed laser deposition (PLD) system. The STO are (100) oriented unless otherwise noted. The deposition temperature $\left(T_{d}\right)$ of $a$ - $\mathrm{LAO}$ is $25^{\circ} \mathrm{C}$ and oxygen partial pressure is $P_{\mathrm{O}_{2}}=2 \times 10^{-6}$ Torr unless otherwise noted. Before deposition, patterns with a Hall bar and a lateral gate pad were fabricated on STO by photolithography [Fig. S1(a)] [54]. The gate electrode was formed by covering silver paint on the lateral gate pad. The wire connection for transport measurement was done by Al ultrasonic wire bonding. A small drop of the IL, $N, N$-diethyl- $N$-methyl- $N$-(2-methoxyethyl)ammonium bis(trifluoromethyl sulphonyl)imide (DEME-TFSI), covered both the channel and gate electrode. The transport measurements were made in Quantum Design Physical Property Measurement System (PPMS), using the built-in Source Measure Units, Keithley 2400 Sourcemeters, and 2002 Multimeters. For the x-ray absorption spectroscopy (XAS) and photoluminescence (PL) measurements, unpatterned large-area samples were prepared [Fig. S1(b)] [54]. The PL measurements were performed under excitation with a He-Cd laser line $(325 \mathrm{~nm})$ and the spectra were collected by iHR 550 spectrometer. The XAS measurements were performed using the Surface, Interface, and Nanostructure Science beam line in the Singapore Synchrotron Light Source. The incident $\mathrm{x}$ ray is normal to the sample surfaces.

Figure 1(a) shows the resistance as a function of $V_{G}$ for a device over ten scan cycles [see Fig. S1(c) for the schematic of an EDLT device] [54]. The device could be switched between low and high-resistance states with hysteresis and irreversibility. The irreversible behavior is more obvious in the first cycle. After the 3rd cycle, the scanning is reversible, even though a slight hysteresis is still observed [inset of Fig. 1(a)]. For comparison, Fig. 1(b) shows the resistance of an oxygen-annealed crystalline LAO/STO device in which $\mathrm{O}_{\text {vac }}$ are absent [52]. A reversible and nonhysteretic metalinsulator transition is observed, indicating pure electrostatic charging and depletion [36]. Moreover, the $a$-LAO/STO remains insulating even when the $V_{G}$ is reversed back to $0 \mathrm{~V}$. These results indicate that the electrostatic effect is not the only mechanism of IL gating on the $a$-LAO/STO interface.
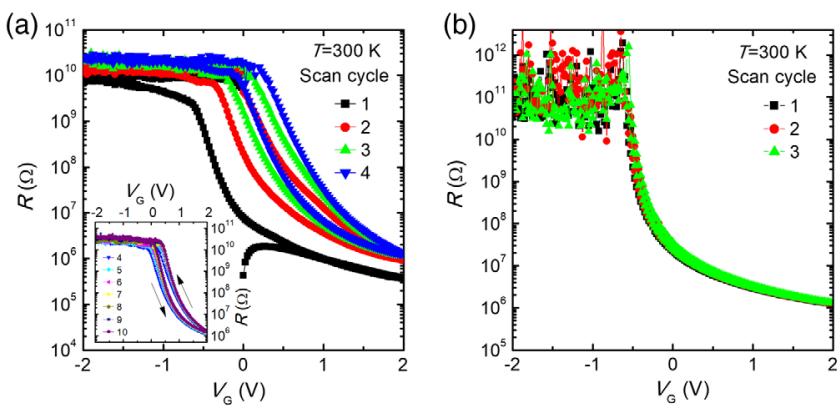

FIG. 1. (a) Resistance $R=V_{\mathrm{SD}} / I_{\mathrm{SD}}$, where $\mathrm{V}_{\mathrm{SD}}$ is the sourcedrain voltage and $\mathrm{I}_{\mathrm{SD}}$ is the source-drain current, as a function of $V_{G}$ for a 2.5 -nm $a$-LAO/STO device. Inset of (a) is the same scanning from 4th to 10th cycles. At each cycle, the scanning of $V_{G}$ starts from 0 to +2 to $-2 \mathrm{~V}$ and stops at $0 \mathrm{~V}$. (b) Resistance as a function of $V_{G}$ for an oxygen-annealed crystalline LAO/STO device. The $V_{G}$ is swept at a speed of $25 \mathrm{mV} / \mathrm{s}$ and $\mathrm{V}_{\mathrm{SD}}=3 \mathrm{~V}$ is applied.

Figures 2(a)-2(c) show sheet resistance $R_{s}$, carrier density $n_{s}$, and Hall mobility $\mu_{H}$ as a function of temperature $T$ for samples before and after gating. The measurements before gating were performed on the pristine device before any IL was applied. The ones after gating were performed after the devices were gated for different cycles ( 1 and 10 cycles) and stopped at different $V_{G}(-2,0$, and $2 \mathrm{~V}$ ), by scanning $V_{G}$ between 2 and $-2 \mathrm{~V}$, and then subsequently removing the IL. One can see that all samples are metallic due to the creation of $\mathrm{O}_{\mathrm{vac}}$ in STO during the LAO deposition [50-52]. However, the samples show higher $R_{s}$ at high $T$ and lower $R_{s}$ at low $T$ after gating, suggesting improved metallic conduction. Moreover, the samples after gating show similar behavior of $R_{s}-T$ curves, even though the samples stopping at $-2 \mathrm{~V}$ show slightly lower $R_{s}$ at low $T$, compared to those stopping at 0 and $2 \mathrm{~V}$.

The sample before gating shows $n_{s}$ of $\sim 1.21 \times 10^{14} \mathrm{~cm}^{-2}$ at $300 \mathrm{~K}$ and $\sim 2.71 \times 10^{13} \mathrm{~cm}^{-2}$ at $4 \mathrm{~K}$. The $n_{s}$ decreases with decreasing $T$ below $\sim 150 \mathrm{~K}$, suggesting carrier freeze out which is characterized by an activation energy $\varepsilon$ of $\sim 5 \mathrm{meV}$ (fitted by $n_{s} \propto e^{-\varepsilon / k_{B} T}$ ) [55], and therefore, the presence of localized electrons. In contrast, $n_{s}$ of the samples after gating is nearly $T$ independent and shows a much lower value of $\sim 2 \times 10^{13} \mathrm{~cm}^{-2}$, suggesting that part of $\mathrm{O}_{\text {vac }}$ was filled. Because of the lower $R_{s}$ and $n_{s}$, the samples after gating show higher $\mu_{H}$ at low $T$ [Fig. 2(c)]. For example, the sample gated for 10 cycles and stopping at $-2 \mathrm{~V}$ shows $\mu_{H}$ of $\sim 1610 \mathrm{~cm}^{2} / \mathrm{V} \mathrm{s}$ at $4 \mathrm{~K}$, higher than that of the sample before gating $\left(\sim 550 \mathrm{~cm}^{2} / \mathrm{V} \mathrm{s}\right)$. The highest $\mu_{H}$ of $\sim 3580 \mathrm{~cm}^{2} / \mathrm{Vs}$ at $3 \mathrm{~K}$ is obtained on the $15-\mathrm{nm}$ $a$-LAO/STO interface, and, consequently, quantum oscillations of the magnetoresistance can be observed (Fig. S2) [54]. Figure 2(d) shows the $R_{s}$ and $n_{s}$ at $300 \mathrm{~K}$ for samples before and after gating ( 10 cycles and stop at $-2 \mathrm{~V}$ ) with different $a$-LAO thickness. We also performed the gating on $a$-LAO/STO interfaces with different STO orientations, 

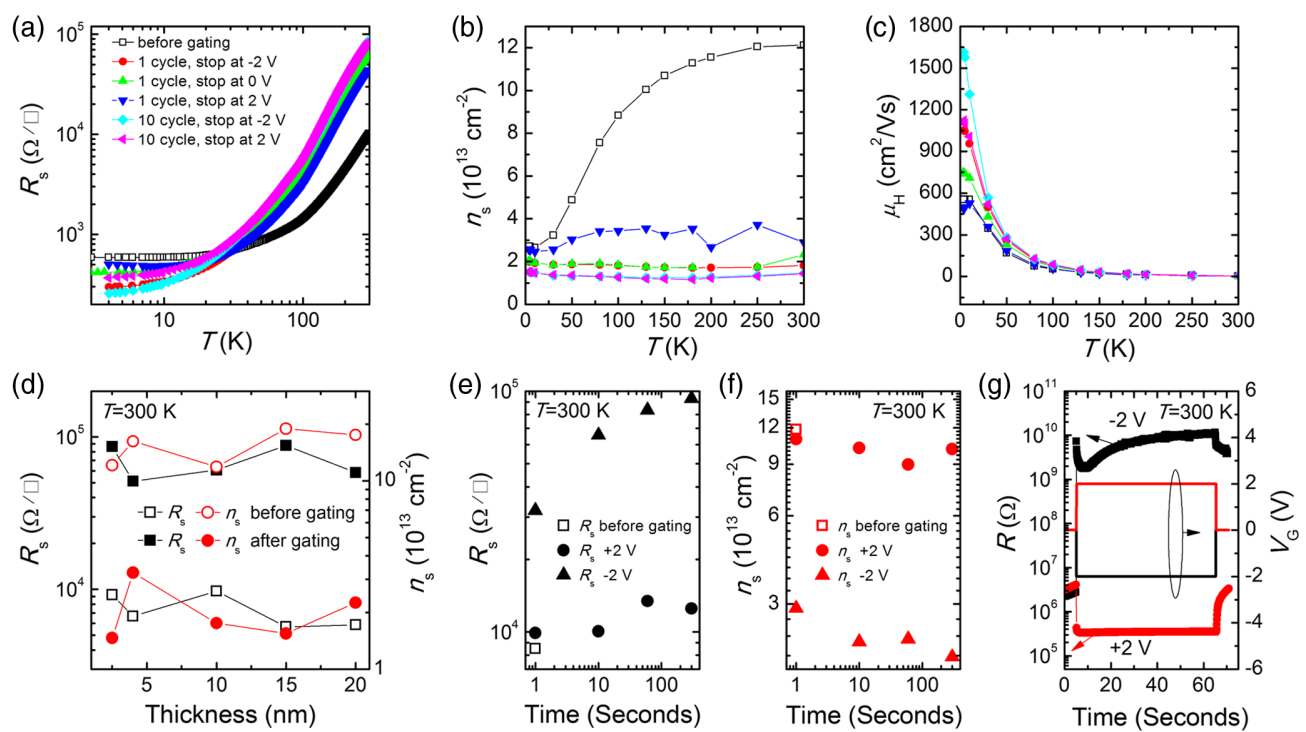

FIG. 2. (a) The $R_{s}-T$ curves of $2.5-\mathrm{nm} a$-LAO/STO samples before and after gating. (b) The $n_{s}$ and (c) $\mu_{H}$ as a function of temperature. (d) $R_{s}$ and $n_{s}$ for samples with $a$-LAO at different thickness. (e) $R_{s}$ and (f) $n_{s}$ for four samples before and after application of one single $V_{G}$ pulse for different duration, $V_{G}=-2$ and $+2 \mathrm{~V}$ for 1, 10, 60, and 300 sec. For convenient comparison, the data before gating is labeled at $1 \mathrm{sec}$. $(\mathrm{g})$ Resistance as a function of time for one single $60-\mathrm{sec} V_{G}$ pulse at -2 and $+2 \mathrm{~V}$.

and interfaces between STO substrates and a series of other amorphous materials (Figs. S3 and S4) [54]. All samples after gating show one order of magnitude higher $R_{s}$ and lower $n_{s}$, further suggesting the universal decrease in $\mathrm{O}_{\text {vac }}$.

Figures 2(e) and 2(f) show the $R_{s}$ and $n_{s}$ for samples before and after application of one single $V_{G}$ pulse for different durations. The $R_{s}$ and $n_{s}$ of samples after $V_{G}=$ $+2 \mathrm{~V}$ pulses are almost unchanged, compared with the sample before gating. In contrast, the samples after $-2 \mathrm{~V}$ pulses show 1 order of magnitude higher $R_{s}$ and lower $n_{s}$. An example of resistance as a function of time for one single 60-sec gate pulse is shown in Fig. 2(g). These results indicate that oxygen filling can be induced only after application of negative $V_{G}$ which causes the samples to be insulating states. Even with a $-2 \mathrm{~V}$ pulse duration of only $1 \mathrm{sec}$, a large change of $R_{s}$ and $n_{s}$ was still observed, suggesting that the filling of $\mathrm{O}_{\mathrm{vac}}$ is a transient process.

Figure 3(a) shows the room-temperature Ti $L_{2,3}$-edge XAS spectra of 2.5-nm $a$-LAO/STO interfaces. In oxygendeficient STO, charge transfer from $\mathrm{O}_{\text {vac }}$ to $\mathrm{Ti}$ atoms, causes a change of $\mathrm{Ti}$ oxidation from $\mathrm{Ti}^{4+}$ to $\mathrm{Ti}^{3+}$, and therefore, the corresponding vacancy concentration can be derived. As indicated by the arrow, the intensity of $458 \mathrm{eV}$ emission, which comes from the $\mathrm{Ti}^{3+} L_{3}$ edge [56,57], decreases after gating. This means that the amount of $\mathrm{Ti}^{3+}$, and therefore the number of $\mathrm{O}_{\mathrm{vac}}$ decrease after gating. The decrease in $\mathrm{O}_{\text {vac }}$ can also be proved from the $\mathrm{O} K$-edge spectra in which the peak intensity arising from Ti-O $p d$ hybridization decreases after gating (Fig. S5) [54,56]. Figure 3(b) shows the room-temperature PL spectra. One can see a broad emission centered at $\sim 430 \mathrm{~nm}$, which is similar to that observed in $\mathrm{Ar}^{+}$-irradiated STO and is caused by the radiative process between the doped conduction electrons and the in-gap state [58], indicating that the emission here originates from the oxygen-deficient STO below $a$-LAO [52,58]. The PL intensity decreases after gating further indicates the decrease in $\mathrm{O}_{\text {vac }}$ concentration. Furthermore, the in situ PL showed that with decreasing $V_{G}$, the intensity decreases continuously, directly indicating oxygen electromigration and the resultant decrease in $\mathrm{O}_{\text {vac }}$ during the gating process (Fig. S5) [54].

Figures 4(a) and 4(b) show the $n_{s}$ and $R_{s}$ at $300 \mathrm{~K}$ before and after gating for LAO/STO interfaces with $T_{d}$ from 25 to $780^{\circ} \mathrm{C}$. All samples were deposited at the same $P_{O 2}=$ $1 \times 10^{-4}$ Torr without post oxygen annealing, and therefore, $\mathrm{O}_{\text {vac }}$ which dominate the charge carriers are present [52]. The pristine interfaces show similar $n_{s}$ of $\sim 1 \times 10^{14} \mathrm{~cm}^{-2}$ and $R_{s}$ of $\sim 10000 \Omega / \square$. After gating, the samples show a general decrease in $n_{s}$ and increase in $R_{s}$ with decreasing $T_{d}$ below
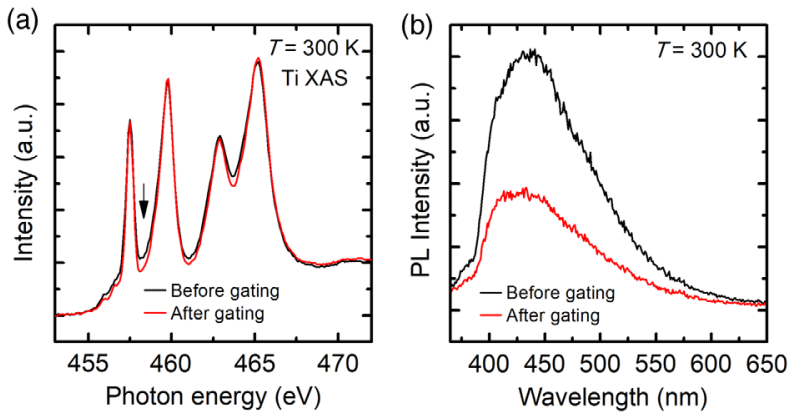

FIG. 3. (a) Ti $L_{2.3}$-edge XAS and (b) PL spectra for 2.5-nm $a$-LAO/STO interfaces before and after gating. The black arrow indicates the emission coming from $\mathrm{Ti}^{3+}$. 

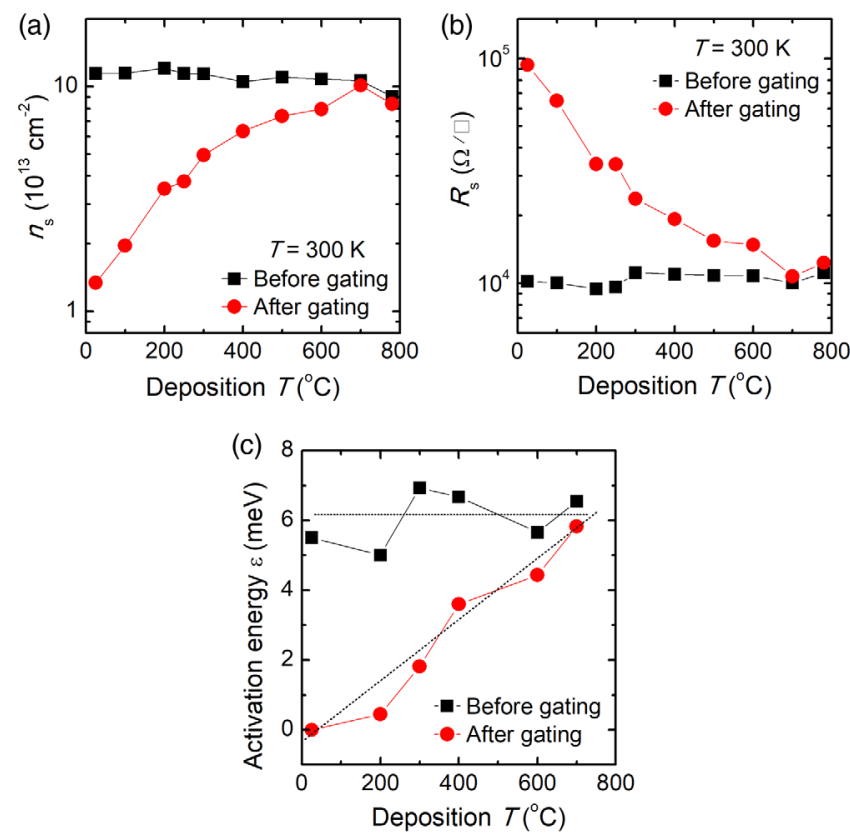

FIG. 4. (a) The $n_{s}$ and (b) $R_{s}$ as a function of $T_{d}$ for samples before and after application of a negative $V_{G}$ pulse for duration of 2 min. (c) The $\varepsilon$ as a function of $T_{d}$. The dot lines are the guides for eyes.

$700{ }^{\circ} \mathrm{C}$. For $T_{d}$ above $500{ }^{\circ} \mathrm{C}$ at which LAO is crystalline [59], samples grown at 780 and $700{ }^{\circ} \mathrm{C}$ show almost unchanged $n_{s}$ and $R_{s}$ after gating, and samples grown at relative low $T_{d}$ of 600 and $500^{\circ} \mathrm{C}$ show slight decrease in $n_{s}$ and increase in $R_{s}$. For $T_{d}$ below $500^{\circ} \mathrm{C}$ at which LAO is amorphous [59], the changes of $n_{s}$ and $R_{s}$ after gating are more obvious with decreasing $T_{d}$, and 1 order of magnitude change is observed at $25^{\circ} \mathrm{C}$. These suggest that more $\mathrm{O}_{\text {vac }}$ were filled at interfaces with lower $T_{d}$, and thus, the filling effect could be controlled by $T_{d}$.

The $n_{s}$ as a function of $T$ for LAO/STO interfaces with different $T_{d}$ were also measured (Fig. S6) [54]. The $\varepsilon$ before and after gating for each sample is shown in Fig. 4(c). Before gating, $n_{s}$ of all samples show similar carrier freeze-out behavior and the $\varepsilon$ are similar ranging from 5 to $7 \mathrm{meV}$. In contrast, the samples show less carrier freeze-out effect after gating with decreasing $T_{d}$, and for $T_{d}=25^{\circ} \mathrm{C}$, the $n_{s}$ is independence of $T$. Correspondingly, the $\varepsilon$ decreases from $\sim 6$ to $0 \mathrm{meV}$ as the $T_{d}$ decreases from 700 to $25^{\circ} \mathrm{C}$.

Figure 5 shows a schematic band diagram of the LAO/STO interface [60-63]. The carriers are thermally activated from $\mathrm{O}_{\mathrm{vac}}$ donor level $\left(E_{\mathrm{Ov}}\right)$ to conduction band $\left(E_{\mathrm{C}}\right)$ and accumulate at the interface at high $T$. As $T$ decreases, more and more free electrons freeze and are trapped at $E_{\mathrm{Ov}}$, and therefore, a carrier freeze-out effect is observed in the samples before gating [Fig. 5(a) and Fig. S6]. After gating, the carrier behavior is the same as that before gating for the interface grown at $700{ }^{\circ} \mathrm{C}$, since the filling is negligible and $E_{\mathrm{Ov}}$ is unchanged [Fig. 5(a)]. For the interface

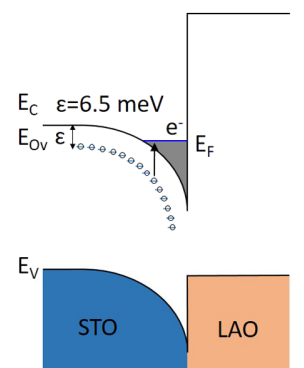

(a)

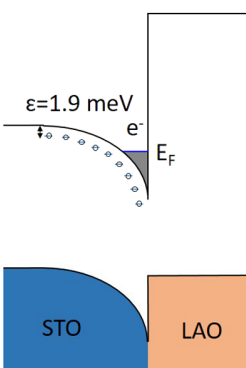

(b)

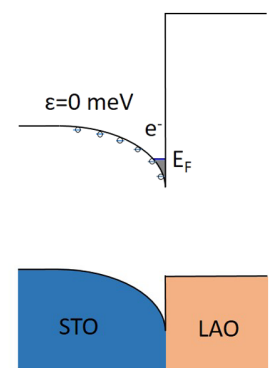

(c)
FIG. 5. Schematic band diagrams of the LAO/STO interfaces after gating for $T_{d}$ of (a) 700 , (b) 300 , and (c) $25^{\circ} \mathrm{C}$. For the interfaces before gating, the band diagrams are the same as shown in (a), since the $R_{s}$ and $n_{s}$ are the same as those after gating at $700{ }^{\circ} \mathrm{C}$. The single arrow in (a) denotes the transition of electrons from $E_{\mathrm{Ov}}$ to $E_{\mathrm{C}}$ as a result of thermal activation. The short lines in the band gap denotes the $E_{\mathrm{Ov}}$ and the circles denote the holes at $E_{\mathrm{Ov}}$ after the electron transition to $E_{\mathrm{C}}$. The $\varepsilon$ after gating are also shown.

grown at $300^{\circ} \mathrm{C}$, some $\mathrm{O}_{\text {vac }}$ are irreversibly filled and $E_{\mathrm{Ov}}$ move closer to $E_{\mathrm{C}}$ after gating [Fig. 5(b)], and thus the $n_{s}$ decreases and shows less carrier freeze-out effect (Fig. S6). For the interface grown at $25^{\circ} \mathrm{C}$, more $\mathrm{O}_{\text {vac }}$ are filled and $E_{\mathrm{Ov}}$ moves to the same level as $E_{\mathrm{C}}$ after gating [Fig. 5(c)]. The free electrons are present at $E_{\mathrm{C}}$ rather than trapped at $E_{\mathrm{Ov}}$ as $T$ decreases, and thus, the carrier freeze-out effect is absent (Fig. S6). Since the $n_{s}$ decreases after filling, the Fermi level $\left(E_{F}\right)$ shifts down and the bands at the STO side show less bending. Therefore, the residual $E_{\mathrm{Ov}}$ after filling are much closer to $E_{\mathrm{C}}$ with decreasing $T_{d}$ and the decrease in $\varepsilon$ is observed, indicating that the residual $E_{\mathrm{Ov}}$ are reconstructed and the reconstruction effect strongly depends on $T_{d}$. After filling, the residual electrons can be induced and depleted reversibly [inset of Fig. 1(a)]. Moreover, after removing the IL, the samples show similar behavior of $R_{s}$ and $n_{s}$, regardless of the $V_{G}$ at which the scanning stops [Figs. 2(a) and 2(b)]. This is different from the observation in $\mathrm{VO}_{2}$, the sample maintained its state at the $V_{G}$ where the scanning stopped [15]. These probably suggest that after partial filling of $\mathrm{O}_{\mathrm{vac}}$, the gating effect on the residual carriers is mainly a result of electrostatic charging and depleting.

The deposited overlayer appears to play an important role in the oxygen filling. It has been found that the filling of $\mathrm{O}_{\mathrm{vac}}$ is not obvious when the LAO layer is crystalline (Fig. 4). We also found that gating performed in the PPMS chamber where oxygen is absent and in air show similar results, suggesting that the filling is not dependent on the oxygen gas environment. Therefore, we deduce that it is the oxygen in the amorphous overlayer that migrates into STO to fill the vacancies, under the influence of electric field [Fig. S1(d)] [54]. Note that the occurrence of $\mathrm{O}_{\text {vac }}$ in LAO can be compensated by a charged interface [47]. Since the filling causes the change of $\mathrm{Ti}$ valence from $\mathrm{Ti}^{3+}$ to $\mathrm{Ti}^{4+}$, the redox reaction during gating process is expected. It has been reported that the surfaces show considerable damage 
after the samples (single crystalline $\mathrm{TiO}_{2}, \mathrm{STO}$, and $\mathrm{La}_{0.5} \mathrm{Sr}_{0.5} \mathrm{CoO}_{3-x}$ ) were irreversibly gated from insulator to metal, due to the electrochemical redox reaction on the sample surfaces $[18,23,64]$. In the present results, the atomic force microscopy images of the sample surfaces before and after gating are essentially unchanged (Fig. S7) [54]. Moreover, the leakage current is negligibly small, on the order of $10^{-9} \mathrm{~A}$. These suggest that the redox reaction only occurs on the STO near the interface, not on the LAO surface or within the electrolyte.

In conclusion, we have demonstrated the electrolyte gating-induced oxygen migration and the resultant filling of $\mathrm{O}_{\text {vac }}$ at LAO/STO interfaces. The filling effect caused the reconstruction of interfacial band structures, which could be controlled by the overlayer deposition temperature, suggesting a method for tuning band structures and oxidation states on buried oxides. Since the filling occurs on the substrate surface, our findings would also be useful for future exploration of electrolyte gating on various emergent oxide interfaces $[65,66]$ and interfaces based on oxide substrates such as superconducting FeSe/STO [67]. Moreover, enhanced carrier mobility and quantum magnetoresistance oscillations are achieved as a result of gating-induced oxygen filling, opening an avenue to construct high-mobility oxide heterostructures which are synthesized at room temperature.

This work is supported by the National University of Singapore (NUS) Academic Research Fund (AcRF Tier 1 Grants No. R-144-000-364-112, No. R-144-000-391-114, and No. R-144-000-403-114) and the Singapore National Research Foundation (NRF) under the Competitive Research Programs (CRP Grant No. NRF-CRP15-201501). P. Y. thanks the support from Singapore Synchrotron Light Source (SSLS) via NUS Core Support C-380-003003-001.

*To whom correspondence should be addressed. phyzen@nus.edu.sg †ariando@nus.edu.sg

[1] C. H. Ahn, A. Bhattacharya, M. Di Ventra, J. N. Eckstein, C. D. Frisbie, M. E. Gershenson, A. M. Goldman, I. H. Inoue, J. Mannhart, A. J. Millis, A. F. Morpurgo, D. Natelson, and J. M. Triscone, Rev. Mod. Phys. 78, 1185 (2006).

[2] H. T. Yuan, H. Shimotani, A. Tsukazaki, A. Ohtomo, M. Kawasaki, and Y. Iwasa, Adv. Funct. Mater. 19, 1046 (2009).

[3] S. Z. Bisri, S. Shimizu, M. Nakano, and Y. Iwasa, Adv. Mater. 29, 1607054 (2017).

[4] Y. Saito, T. Nojima, and Y. Iwasa, Nat. Rev. Mater. 2, 16094 (2016).

[5] K. Ueno, S. Nakamura, H. Shimotani, A. Ohtomo, N. Kimura, T. Nojima, H. Aoki, Y. Iwasa, and M. Kawasaki, Nat. Mater. 7, 855 (2008).

[6] J. T. Ye, Y. J. Zhang, R. Akashi, M. S. Bahramy, R. Arita, and Y. Iwasa, Science 338, 1193 (2012).
[7] S. Jo, D. Costanzo, H. Berger, and A. F. Morpurgo, Nano Lett. 15, 1197 (2015).

[8] J. Lu, O. Zheliuk, Q. Chen, I. Leermakers, N. E. Hussey, U. Zeitler, and J. Ye, Proc. Natl. Acad. Sci. U.S.A. 115, 3551 (2018).

[9] X. X. Xi, H. Berger, L. Forro, J. Shan, and K. F. Mak, Phys. Rev. Lett. 117, 106801 (2016).

[10] Y. Saito, Y. Kasahara, J. T. Ye, Y. Iwasa, and T. Nojima, Science 350, 409 (2015).

[11] Y. Saito, T. Nojima, and Y. Iwasa, Nat. Commun. 9, 778 (2018).

[12] Y. Yu, F. Yang, X. F. Lu, Y. J. Yan, Y. H. Cho, L. Ma, X. Niu, S. Kim, Y. W. Son, D. Feng, S. Li, S. W. Cheong, X. H. Chen, and Y. Zhang, Nat. Nanotechnol. 10, 270 (2015).

[13] M. Nakano, K. Shibuya, D. Okuyama, T. Hatano, S. Ono, M. Kawasaki, Y. Iwasa, and Y. Tokura, Nature (London) 487, 459 (2012).

[14] S. W. Zeng, Z. Huang, W. M. Lv, N. N. Bao, K. Gopinadhan, L. K. Jian, T. S. Herng, Z. Q. Liu, Y. L. Zhao, C. J. Li, H. J. H. Ma, P. Yang, J. Ding, T. Venkatesan, and Ariando, Phys. Rev. B 92, 020503 (2015).

[15] J. Jeong, N. Aetukuri, T. Graf, T. D. Schladt, M. G. Samant, and S. S. P. Parkin, Science 339, 1402 (2013).

[16] N. Lu, P. Zhang, Q. Zhang, R. Qiao, Q. He, H. B. Li, Y. Wang, J. Guo, D. Zhang, Z. Duan, Z. Li, M. Wang, S. Yang, M. Yan, E. Arenholz, S. Zhou, W. Yang, L. Gu, C. W. Nan, J. Wu et al., Nature (London) 546, 124 (2017).

[17] T. A. Petach, A. Mehta, R. Marks, B. Johnson, M. F. Toney, and D. Goldhaber-Gordon, ACS Nano 10, 4565 (2016).

[18] T. D. Schladt, T. Graf, N. B. Aetukuri, M. Y. Li, A. Fantini, X. Jiang, M. G. Samant, and S. S. P. Parkin, ACS Nano 7, 8074 (2013).

[19] M. Y. Li, W. Han, X. Jiang, J. Jeong, M. G. Samant, and S. S. P. Parkin, Nano Lett. 13, 4675 (2013).

[20] S. Chen, X. j. Wang, L. Fan, G. Liao, Y. Chen, W. Chu, L. Song, J. Jiang, and C. Zou, Adv. Funct. Mater. 26, 3532 (2016).

[21] T. A. Petach, M. Lee, R. C. Davis, A. Mehta, and D. Goldhaber-Gordon, Phys. Rev. B 90, 081108 (2014).

[22] P. Xu, W. Han, P. M. Rice, J. Jeong, M. G. Samant, K. Mohseni, H. L. Meyerheim, S. Ostanin, I. V. Maznichenko, I. Mertig, E. K. Gross, A. Ernst, and S. S. Parkin, Adv. Mater. 29, 1604447 (2017).

[23] J. Walter, H. Wang, B. Luo, C. D. Frisbie, and C. Leighton, ACS Nano 10, 7799 (2016).

[24] P. R. Pudasaini, J. H. Noh, A. T. Wong, O. S. Ovchinnikova, A. V. Haglund, S. Dai, T. Z. Ward, D. Mandrus, and P. D. Rack, Adv. Funct. Mater. 26, 2820 (2016).

[25] A. M. Perez-Muñoz, P. Schio, R. Poloni, A. FernandezMartinez, A. Rivera-Calzada, J. C. Cezar, E. Salas-Colera, G. R. Castro, J. Kinney, C. Leon, J. Santamaria, J. GarciaBarriocanal, and A. M. Goldman, Proc. Natl. Acad. Sci. U.S.A. 114, 215 (2017).

[26] H. T. Yi, B. Gao, W. Xie, S. W. Cheong, and V. Podzorov, Sci. Rep. 4, 6604 (2014).

[27] H. Ji, J. Wei, and D. Natelson, Nano Lett. 12, 2988 (2012).

[28] K. Shibuya and A. Sawa, Adv. Electron. Mater. 2, 1500131 (2016). 
[29] H. T. Yuan, H. Shimotani, A. Tsukazaki, A. Ohtomo, M. Kawasaki, and Y. Iwasa, J. Am. Chem. Soc. 132, 6672 (2010).

[30] D. Okuyama, M. Nakano, S. Takeshita, H. Ohsumi, S. Tardif, K. Shibuya, T. Hatano, H. Yumoto, T. Koyama, H. Ohashi, M. Takata, M. Kawasaki, T. Arima, Y. Tokura, and Y. Iwasa, Appl. Phys. Lett. 104, 023507 (2014).

[31] J. Jeong, N. B. Aetukuri, D. Passarello, S. D. Conradson, M. G. Samant, and S. S. P. Parkin, Proc. Natl. Acad. Sci. U.S.A. 112, 1013 (2015).

[32] G. Dubuis, Y. Yacoby, H. Zhou, X. He, A. T. Bollinger, D. Pavuna, R. Pindak, and I. Bozovic, Sci. Rep. 6, 32378 (2016).

[33] D. Passarello, S. G. Altendorf, J. Jeong, M. G. Samant, and S. S. Parkin, Nano Lett. 16, 5475 (2016).

[34] Y. Zhou, J. Park, J. Shi, M. Chhowalla, H. Park, D. A. Weitz, and S. Ramanathan, Nano Lett. 15, 1627 (2015).

[35] P. Gallagher, M. Lee, T. A. Petach, S. W. Stanwyck, J. R. Williams, K. Watanabe, T. Taniguchi, and D. GoldhaberGordon, Nat. Commun. 6, 6437 (2015)

[36] S. W. Zeng, W. M. Lu, Z. Huang, Z. Q. Liu, K. Han, K. Gopinadhan, C. J. Li, R. Guo, W. X. Zhou, H. J. H. Ma, L. K. Jian, T. Venkatesan, and Ariando, ACS Nano 10, 4532 (2016).

[37] W. N. Lin, J. F. Ding, S. X. Wu, Y. F. Li, J. Lourembam, S. Shannigrahi, S. J. Wang, and T. Wu, Adv. Mater. Interfaces 1, 1300001 (2013).

[38] Z. Chen, H. Yuan, Y. Xie, D. Lu, H. Inoue, Y. Hikita, C. Bell, and H. Y. Hwang, Nano Lett. 16, 6130 (2016).

[39] A. Ohtomo and H. Y. Hwang, Nature (London) 427, 423 (2004).

[40] N. Reyren, S. Thiel, A. D. Caviglia, L. F. Kourkoutis, G. Hammerl, C. Richter, C. W. Schneider, T. Kopp, A. S. Ruetschi, D. Jaccard, M. Gabay, D. A. Muller, J. M. Triscone, and J. Mannhart, Science 317, 1196 (2007).

[41] A. Brinkman, M. Huijben, M. Van Zalk, J. Huijben, U. Zeitler, J. C. Maan, W. G. Van der Wiel, G. Rijnders, D. H. A. Blank, and H. Hilgenkamp, Nat. Mater. 6, 493 (2007).

[42] A. D. Caviglia, S. Gariglio, N. Reyren, D. Jaccard, T. Schneider, M. Gabay, S. Thiel, G. Hammerl, J. Mannhart, and J. M. Triscone, Nature (London) 456, 624 (2008).

[43] Ariando, X. Wang, G. Baskaran, Z. Q. Liu, J. Huijben, J. B. Yi, A. Annadi, A. R. Barman, A. Rusydi, S. Dhar, Y. P. Feng, J. Ding, H. Hilgenkamp, and T. Venkatesan, Nat. Commun. 2, 188 (2011).

[44] Z. Huang, K. Han, S. Zeng, M. Motapothula, A. Y. Borisevich, S. Ghosh, W. Lu, C. Li, W. Zhou, Z. Liu, M. Coey, T. Venkatesan, and Ariando, Nano Lett. 16, 2307 (2016).

[45] Y. W. Xie, Y. Hikita, C. Bell, and H. Y. Hwang, Nat. Commun. 2, 494 (2011).

[46] D. C. Vaz, E. Lesne, A. Sander, H. Naganuma, E. Jacquet, J. Santamaria, A. Barthelemy, and M. Bibes, Adv. Mater. 29, 1700486 (2017).

[47] L. Yu and A. Zunger, Nat. Commun. 5, 5118 (2014).

[48] C. Cen, S. Thiel, and J. Mannhart, Science 323, 1026 (2009).

[49] J. Mannhart and D. G. Schlom, Science 327, 1607 (2010).
[50] Y. Z. Chen, N. Pryds, J. E. Kleibeuker, G. Koster, J. R. Sun, E. Stamate, B. G. Shen, G. Rijnders, and S. Linderoth, Nano Lett. 11, 3774 (2011).

[51] S. W. Lee, Y. Q. Liu, J. Heo, and R. G. Gordon, Nano Lett. 12, 4775 (2012).

[52] Z. Q. Liu, C. J. Li, W. M. Lu, X. H. Huang, Z. Huang, S. W. Zeng, X. P. Qiu, L. S. Huang, A. Annadi, J. S. Chen, J. M. D. Coey, T. Venkatesan, and Ariando, Phys. Rev. X 3, 021010 (2013).

[53] Y.Z. Chen et al., Nat. Mater. 14, 801 (2015).

[54] See Supplemental Material at http://link.aps.org/ supplemental/10.1103/PhysRevLett.121.146802 for the following: Fig. S1 for the schematic of EDLT operation, schematic of oxygen filling on the STO surface and optical micrograph of EDLT device pattern; Fig. S2 for the observation of $\mathrm{SdH}$ oscillation at $a$-LAO/STO interface after gating; Figs. S3 and $\mathrm{S} 4$ for $R_{s}$ and $n_{s}$ of $a$-LAO/STO interfaces with different STO orientations, and interfaces between STO substrates and a series of other amorphous materials, before and after gating; Fig. S5 for the $\mathrm{O} K$-edge XAS spectra and PL spectra for $a$-LAO/STO interfaces before and after gating; Fig. S6 for the temperature dependent $n_{s}$ of samples before and after gating, with different LAO overlayer deposition temperature; Fig. S7 for AFM images of the sample surfaces before and after gating experiments.

[55] Z. Q. Liu, D. P. Leusink, X. Wang, W. M. Lu, K. Gopinadhan, A. Annadi, Y. L. Zhao, X. H. Huang, S. W. Zeng, Z. Huang, A. Srivastava, S. Dhar, T. Venkatesan, and Ariando, Phys. Rev. Lett. 107, 146802 (2011).

[56] C. Y. Chen, J. Avila, E. Frantzeskakis, A. Levy, and M. C. Asensio, Nat. Commun. 6, 8585 (2015).

[57] K. Han, N. Palina, S. W. Zeng, Z. Huang, C. J. Li, W. X. Zhou, D. Y. Wan, L. C. Zhang, X. Chi, R. Guo, J. S. Chen, T. Venkatesan, A. Rusydi, and Ariando, Sci. Rep. 6, 25455 (2016).

[58] D. S. Kan, T. Terashima, R. Kanda, A. Masuno, K. Tanaka, S. C. Chu, H. Kan, A. Ishizumi, Y. Kanemitsu, Y. Shimakawa, and M. Takano, Nat. Mater. 4, 816 (2005).

[59] C. Li, Z. Liu, W. Lu, X. R. Wang, A. Annadi, Z. Huang, S. Zeng, Ariando, and T. Venkatesan, Sci. Rep. 5, 13314 (2015).

[60] K. Yoshimatsu, R. Yasuhara, H. Kumigashira, and M. Oshima, Phys. Rev. Lett. 101, 026802 (2008).

[61] B. C. Huang, Y. P. Chiu, P. C. Huang, W. C. Wang, V. T. Tra, J. C. Yang, Q. He, J. Y. Lin, C. S. Chang, and Y. H. Chu, Phys. Rev. Lett. 109, 246807 (2012).

[62] G. De Luca, A. Rubano, E. d. Gennaro, A. Khare, F. M. Granozio, U. S. di Uccio, L. Marrucci, and D. Paparo, Appl. Phys. Lett. 104, 261603 (2014).

[63] A. E. M. Smink, J. C. de Boer, M. P. Stehno, A. Brinkman, W. G. van der Wiel, and H. Hilgenkamp, Phys. Rev. Lett. 118, 106401 (2017).

[64] K. Ueno, H. Shimotani, Y. Iwasa, and M. Kawasaki, Appl. Phys. Lett. 96, 252107 (2010).

[65] S. Stemmer and S. J. Allen, Annu. Rev. Mater. Res. 44, 151 (2014).

[66] J. Chakhalian, J. W. Freeland, A. J. Millis, C. Panagopoulos, and J. M. Rondinelli, Rev. Mod. Phys. 86, 1189 (2014).

[67] J. Shiogai, Y. Ito, T. Mitsuhashi, T. Nojima, and A. Tsukazaki, Nat. Phys. 12, 42 (2016). 